\title{
- Steerable Hopping Six-Legged Robot
}

Motions of spring legs are coordinated in both launch and landing.

NASA's Jet Propulsion Laboratory, Pasadena, California

The figure depicts selected aspects of a six-legged robot that moves by hopping and that can be steered in the sense that it can be launched into a hop in a controllable direction. This is a prototype of hopping robots being developed for use in scientific exploration of rough terrain on remote planets that have surface gravitation less than that of Earth. Hopping robots could also be used on Earth, albeit at diminished hopping distances associated with the greater Earth gravitation.

The upper end of each leg is connected through two universal joints to an upper and a lower hexagonal frame, such that the tilt of the leg depends on the relative position of the two frames. Two non-backdriveable worm-gear motor drives are used to control the relative position of the two frames along two axes $120^{\circ}$ apart, thereby controlling the common tilt of all six legs and thereby, further, controlling the direction of hopping.

Each leg includes an upper and a lower aluminum frame segment with a joint between them. A fiberglass spring, connected via hinges to both segments, is used to store hopping energy prior to launch into a hop and to cushion the landing at the end of the hop. A cable for loading the spring is run into each leg through the center of the universal joints and then down along the center lines of the segments to the lower end of the leg. A central spool actuated by a motor with a harmonic drive and an electromagnetic clutch winds in all six cables to compress all six springs (thereby also flexing all six legs) simultaneously. To ensure that all the legs push off and land in the same direction, timing-belt pulley drives are attached to the leg segments, restricting the flexing and extension of all six legs to a common linear motion.

In preparation for a hop, the spool can be driven to load the spring legs by an amount corresponding to a desired hop distance within range. The amount of compression can be computed from the reading of a shaft-angle

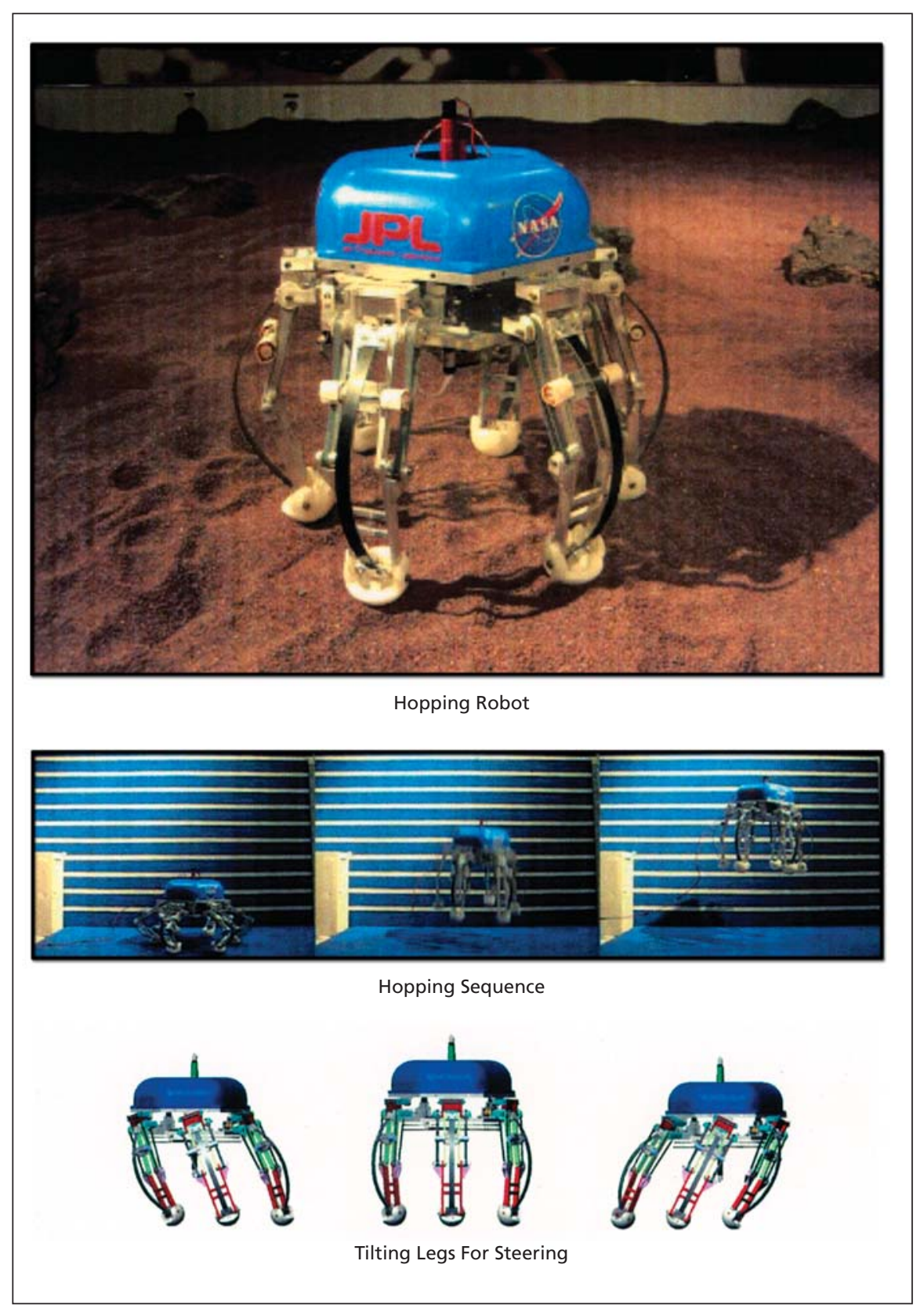

Six Spring Legs are driven in coordinated flexion and extension to effect hopping motion. The legs can be tilted to control the direction of hopping. A gyroscope helps to prevent tumbling during launch, flight, and landing.

encoder that indicates the amount by which the spool has been turned. When the robot is ready to hop, the electromagnetic clutch disengages the motor from the spool, thus releasing the cable restraints on the springs and allowing the springs to extend all six legs simultaneously. 
When the robot lands, the springs in the legs are compressed as they absorb much of the impact. As the legs retract, a constant-force spring motor attached to the spool winds in the leg cables to keep them taught. A unidirectional clutch in line with the spool and the spool motor drive allows the spool to quickly overrun the motor drive when winding up the cable, but locks when the springs in the legs try to pull the cable back out. This action prevents bouncing after landing and provides for storage of energy for reuse on the next hop. A motor-driven gyroscope mounted on the lower hexagonal frame helps to prevent tumbling of the robot during hopping and was tested through computer simulation.

This work was done by Paulo Younse and Hrand Aghazarian of Caltech for NASA's Jet Propulsion Laboratory. Further information is contained in a TSP (see page 1). NPO45062

\section{Launchable and Retrievable Tetherobot}

\section{The reach of an exploratory robot on rough terrain would be extended.}

\section{NASA's Jet Propulsion Laboratory, Pasadena, California}

A proposed robotic system for scientific exploration of rough terrain would include a stationary or infrequently moving larger base robot, to which would be tethered a smaller hopping robot of the type described in the immediately preceding article. The two-robot design would extend the reach of the base robot, making it possible to explore nearby locations that might otherwise be inaccessible or too hazardous for the base robot.

The system would include a launching mechanism and a motor-driven reel on the larger robot. The outer end of the tether would be attached to the smaller robot; the inner end of the tether would be attached to the reel.

The figure depicts the launching and retrieval process. The launching mechanism would aim and throw the smaller robot toward a target location, and the tether would be paid out from the reel as the hopping robot flew toward the target. Upon completion of exploratory activity at the target location, the smaller robot would be made to hop and, in a coordinated motion, the tether would be wound onto the reel to pull the smaller robot back to the larger one.

At the time of reporting the information for this article, the launching and retrieval processes had been studied by computational simulations for various
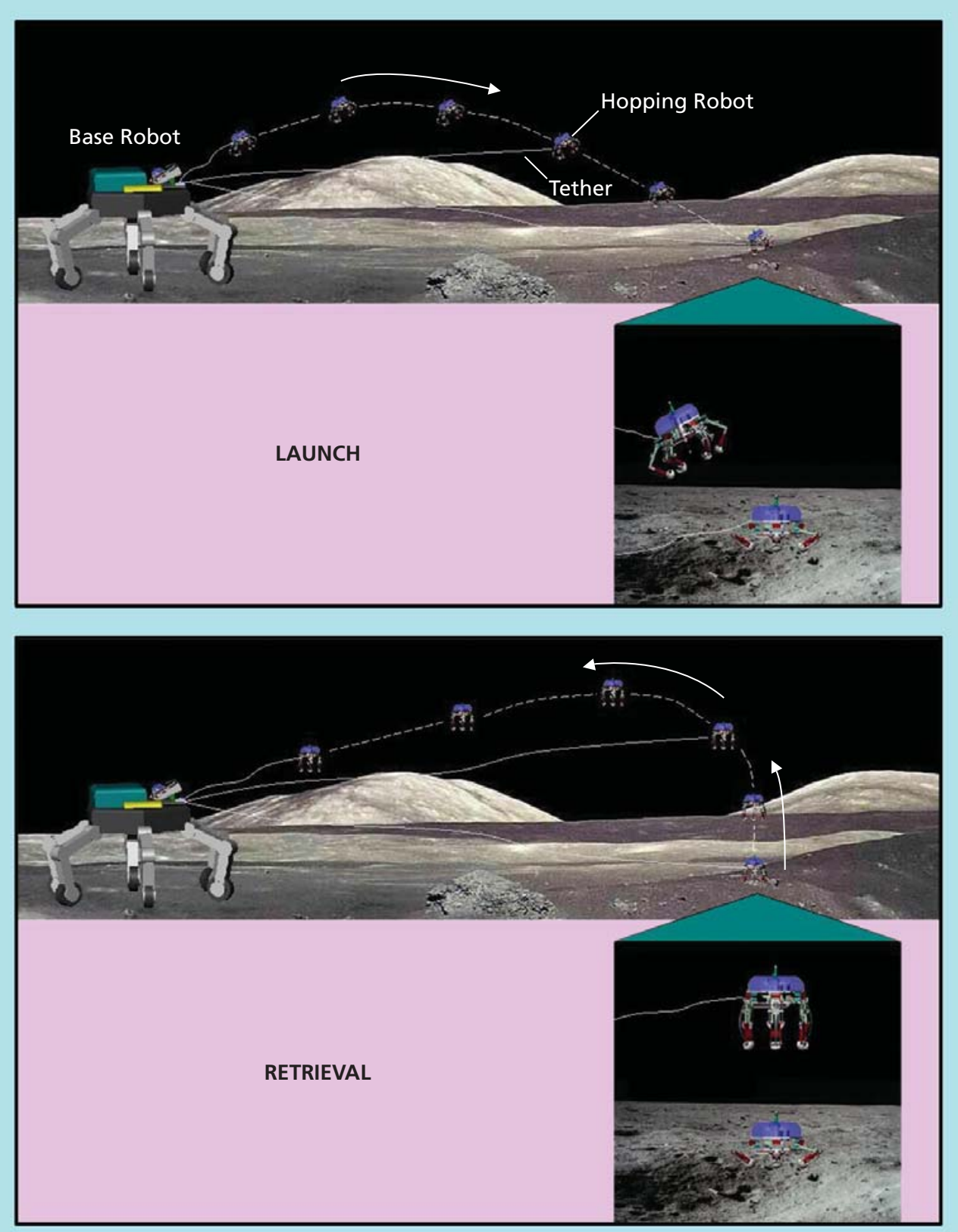

The Smaller Tethered Hopping Robot would be thrown toward a target by a launching mechanism on the larger robot. Later, the hopping robot would be retrieved by reeling in the tether during a hop. 